\title{
Multicenter large-scale study of prognostic impact of HER2 expression in patients with resectable gastric cancer
}

\author{
Yukinori Kurokawa • Nariaki Matsuura • Yutaka Kimura • Shinichi Adachi • \\ Junya Fujita $\cdot$ Hiroshi Imamura $\cdot$ Kenji Kobayashi · Yuhki Yokoyama · \\ Mohammed Nouri Shaker $\cdot$ Shuji Takiguchi $\cdot$ Masaki Mori $\cdot$ Yuichiro Doki
}

Received: 11 June 2014/Accepted: 30 August 2014/Published online: 16 September 2014

(C) The International Gastric Cancer Association and The Japanese Gastric Cancer Association 2014

\begin{abstract}
Background Although some small-scale studies have suggested that human epidermal growth factor receptor 2 (HER2)-positive status in gastric cancer is associated with poor outcomes, the prognostic value of HER2 is still controversial. Since intratumoral HER2 heterogeneity is also an important issue, a multicenter large-scale study was conducted to evaluate the prognostic impacts of HER2 expression and intratumoral heterogeneity in gastric cancer. Methods This study included 1,148 gastric cancer patients who underwent gastrectomy in 11 institutions. HER2 expression was centrally evaluated with
\end{abstract}

Y. Kurokawa ( $₫)$ - S. Takiguchi · M. Mori · Y. Doki Department of Gastroenterological Surgery, Osaka University Graduate School of Medicine, 2-2-E2 Yamadaoka, Suita, Osaka 565-0871, Japan

e-mail: ykurokawa@gesurg.med.osaka-u.ac.jp

N. Matsuura $\cdot$ Y. Yokoyama $\cdot$ M. N. Shaker

Department of Molecular Pathology, Osaka University Graduate

School of Medicine and Health Science, Osaka, Japan

Y. Kimura

Department of Surgery, Sakai Municipal Hospital, Osaka, Japan

S. Adachi

Department of Surgery, Ikeda City Hospital, Osaka, Japan

J. Fujita

Department of Surgery, NTT West Osaka Hospital, Osaka, Japan

H. Imamura

Department of Surgery, Toyonaka Municipal Hospital, Osaka, Japan

K. Kobayashi

Department of Surgery, Kinki Central Hospital, Itami, Hyogo, Japan immunohistochemistry and fluorescence in situ hybridization, and intratumoral HER2 heterogeneity was evaluated for HER2-positive tumors. Overall survival was compared between HER2-positive and HER2-negative patients and between the homogeneous and heterogeneous groups.

Results The HER2-positive rate was $15.7 \%$, and HER2 expression was significantly associated with histological type. HER2 expression scores obtained by immunohistochemistry showed a distinct influence on survival, and HER2-positive patients showed much poorer survival than HER2-negative patients [hazard ratio (HR) 1.59, $95 \%$ confidence interval (CI) 1.24-2.02; $P<0.001$ ). The subgroup analysis by pathological tumor stage showed a similar trend of poor survival in HER2-positive patients. Both intestinal type and diffuse type showed significant poor survival in HER2-positive patients. Cox multivariate analysis revealed that HER2 expression was an independent prognostic factor (HR 1.96, $95 \%$ CI 1.51-2.55; $P<0.001)$. HER2 heterogeneity was observed in $75.4 \%$ of HER2-positive cases, but the prognosis in the heterogeneous group was similar to that in the homogeneous group. Conclusions Our study demonstrated that HER2 overexpression is an independent prognostic factor in patients with any stage of resectable gastric cancer. Intratumoral HER2 heterogeneity did not affect prognosis.

Keywords Human epidermal growth factor receptor 2 . Epidermal growth factor receptor 2 - ERBB2 .

Trastuzumab · Heterogeneity

\section{Introduction}

Gastric cancer remains a major health issue and a frequent cause of cancer death worldwide, although the prevalence 
and mortality of the disease have gradually decreased [1]. In eastern Asia, including Japan, the incidence of gastric cancer is still high despite advances in treatment and subsequent improvements in prognosis. Complete resection of localized tumors is the primary treatment, but recurrence and metastatic spread occur frequently nevertheless. The development of new agents and combination chemotherapies for advanced gastric cancer has led to a steady increase in overall survival (OS). Recently, targeted therapies have significantly impacted the treatment strategy for many common malignancies, including breast, colorectal, and lung cancers. Among these targeted therapies, monoclonal antibody against human epidermal growth factor receptor 2 (HER2) was demonstrated to be highly effective in HER2positive breast cancer, and was approved by the US Food and Drug Administration as the first molecularly targeted agent for solid cancer [2]. In breast cancer, it is well known that HER2 overexpression is a prognostic factor and also a predictive factor for treatment with trastuzumab (antihuman HER2 monoclonal antibody) [3, 4]. In addition, a randomized controlled trial (ToGA study) recently demonstrated that treatment with trastuzumab could extend the OS of patients with HER2-positive gastric cancer [5].

Regarding the relationship between HER2 status and prognosis, some studies have suggested that HER2-positive status in gastric cancer is associated with poor outcomes and aggressive disease [6-8]. However, the sample sizes of these studies were relatively small, and not all studies have shown an association with poor outcomes $[9,10]$, so the prognostic value of HER2 in gastric cancer is still controversial. We therefore conducted a multicenter largescale study to clarify the prognostic impact of HER2 expression in patients with gastric cancer. Furthermore, since intratumoral HER2 heterogeneity is an important issue, especially in gastric cancer [11], we evaluated the clinicopathological characteristics of heterogeneous cases and their impact on prognosis.

\section{Methods}

\section{Patient population}

This study included patients with gastric cancer of any stage who underwent gastrectomy between 2000 and 2006 at any of the 11 institutions of the Osaka University Clinical Research Group for Gastroenterological Surgery. Patients who received chemotherapy or radiation therapy before surgery were excluded. No patients were given trastuzumab, even after recurrence. All tumors were histologically diagnosed as adenocarcinoma of the stomach. Pathological tumor staging was performed according to the seventh edition of the International Union Against Cancer
TNM classification [12]. This study was approved by the institutional review board of each institution involved.

Immunohistochemistry

Archived formalin-fixed, paraffin-embedded specimens were shipped to the institution performing central pathology review. Each specimen was composed of several blocks of tumor oriented longitudinally from proximal to distal stomach, including adjacent normal gastric tissue in each case. Immunohistochemical analysis was carried out on $4-\mu \mathrm{m}$-thick tissue sections. The primary antibody was mouse monoclonal anti-HER2 (clone 4B5, Ventana Medical Systems, Tucson, AZ, USA). Immunostaining was performed using a Ventana BenchMark XT ${ }^{\circledR}$ autoimmunostainer with an iVIEW DAB universal kit (Ventana Medical Systems, Tucson, AZ, USA). Antigen retrieval was performed by autoclaving in EDTA-base buffer, $\mathrm{pH}$ 8.5 at $98{ }^{\circ} \mathrm{C}$ for $60 \mathrm{~min}$. After endogenous peroxidase had been briefly blocked, the sections were incubated with primary antibodies at $37{ }^{\circ} \mathrm{C}$ for $32 \mathrm{~min}$, then incubated with a mixture of goat biotinylated anti-mouse $\mathrm{IgG}$, antimouse IgM, and anti-rabbit IgG antibodies for $8 \mathrm{~min}$ and with peroxidase-conjugated streptavidin for $8 \mathrm{~min}$. Visualization was performed by incubation in 3-diaminobenzidine solution for $8 \mathrm{~min}$, followed by signal amplification with copper sulfate for $4 \mathrm{~min}$ and counterstaining with hematoxylin for $4 \mathrm{~min}$. Specimens of HER2-positive breast cancer were used as a positive control, and slides processed without the primary antibodies were used as negative controls.

\section{Evaluations}

Central pathologists independently performed immunohistochemical analysis without prior knowledge of the patients' clinical data. Immunostaining of the cell membranes of HER2-positive tumors was scored using a fourgrade scale $(0 / 1+/ 2+/ 3+)$ (ToGA score): 0 , no reactivity or membranous reactivity in less than $10 \%$ of tumor cells; $1+$, faint or barely perceptible membranous reactivity in at least $10 \%$ of tumor cells; $2+$, weak to moderate complete, basolateral or lateral membranous reactivity in at least $10 \%$ of tumor cells; and $3+$, strong complete, basolateral or lateral membranous reactivity in at least $10 \%$ of tumor cells. For specimens with an immunohistochemistry (IHC) score of $2+$ only, fluorescence in situ hybridization (FISH) analysis of HER2 status was performed with a Path Vysion HER2 DNA probe kit (Vysis/Abbott, Abbott Park, IL, USA) following the manufacturer's instructions. For FISH, the total numbers of HER2 and chromosome 17 signals were counted in at least 20 tumor cell nuclei in two different areas. The HER2/chromosome 17 ratios were 
interpreted in accordance with the ToGA FISH scoring scheme for HER2 testing in gastric and gastroesophageal junction cancer as follows: less than 2.0, HER2 gene not amplified; 2.0 or more, HER 2 gene amplified. An IHC score of $3+$ or an IHC score of $2+$ with FISH positivity was defined as HER2 positive, whereas IHC scores of 0 or $1+$, or an IHC score of $2+$ with FISH negativity was defined as HER2 negative [5, 11]. Regarding intratumoral heterogeneity of HER2 overexpression, cases with HER2 IHC scores of $2+$ or $3+$ in more than $90 \%$ of tumor cells were considered to be homogeneous, whereas heterogeneous cases were defined as those in which $10-90 \%$ of tumor cells had IHC scores of $2+$ or $3+$.

\section{Statistical analysis}

The relationship between HER2 expression and clinicopathological factors was analyzed using the chi square test for categorical variables and the Mann-Whitney $U$ test for continuous variables. OS was defined as the interval from the date of surgery to the date of death from any cause. Survival rates were estimated using the Kaplan-Meier method and were compared with the log-rank test. The hazard ratio (HR) for death in HER2-positive patients was estimated with a Cox proportional hazard model. Multivariate Cox regression analysis was performed to adjust for potential confounding factors. $P<0.05$ was considered statistically significant. All statistical analyses were performed using SPSS Statistics, version 20 (IBM, Armonk, NY, USA).

\section{Results}

\section{Expression status}

The IHC results for HER2 expression in 1,148 tumors were as follows: score 0, $657(57.2 \%)$; score 1+, 207 $(18.0 \%)$; score $2+, 123(10.7 \%)$; and score $3+, 161$ $(14.0 \%)$. Of the 123 tumors with an IHC score of $2+, 19$ $(15.4 \%)$ were HER2 positive by FISH. In total, 180 (15.7\%) of all 1,148 tumors were diagnosed as HER2 positive. The patients' clinicopathological factors were compared by HER2 status (Table 1). HER2-positive cases were found more frequently in the intestinal type of adenocarcinoma $(P<0.001)$. Tumors located in the upper body of the stomach were more likely to be HER2 positive. The HER2 positivity rates according to the pathological tumor staging were $16.4 \%$ in stage I tumors, $11.8 \%$ in stage II tumors, $13.3 \%$ in stage III tumors, and $24.3 \%$ in stage IV tumors, respectively. The other factors, including $\mathrm{pT}$ and $\mathrm{pN}$ stages, showed no correlation with HER 2 positivity.
Table 1 Characteristics of 1,148 patients

\begin{tabular}{|c|c|c|c|}
\hline Characteristics & $\begin{array}{l}\text { HER2 positive } \\
(n=180)\end{array}$ & $\begin{array}{l}\text { HER2 negative } \\
(n=968)\end{array}$ & $P$ \\
\hline \multicolumn{4}{|l|}{ Age (years) } \\
\hline Median & 67.5 & 67 & \multirow[t]{2}{*}{0.17} \\
\hline Range & $37-87$ & $31-98$ & \\
\hline \multicolumn{4}{|l|}{ Sex } \\
\hline Male & 133 & 657 & \multirow[t]{2}{*}{0.11} \\
\hline Female & 47 & 311 & \\
\hline \multicolumn{4}{|l|}{ Location } \\
\hline Upper & 49 & 206 & \multirow[t]{2}{*}{0.078} \\
\hline $\begin{array}{c}\text { Middle/ } \\
\text { lower }\end{array}$ & 131 & 762 & \\
\hline \multicolumn{4}{|c|}{ Histological type } \\
\hline $\begin{array}{l}\text { Intestinal } \\
\text { type }\end{array}$ & 142 & 478 & \multirow[t]{2}{*}{$<0.001$} \\
\hline Diffuse type & 38 & 490 & \\
\hline \multicolumn{4}{|l|}{$\mathrm{pT}$} \\
\hline $\mathrm{T} 1$ & 76 & 369 & \multirow[t]{4}{*}{0.36} \\
\hline $\mathrm{T} 2$ & 24 & 106 & \\
\hline $\mathrm{T} 3$ & 32 & 220 & \\
\hline $\mathrm{T} 4$ & 48 & 273 & \\
\hline \multicolumn{4}{|l|}{$\mathrm{pN}$} \\
\hline N0 & 90 & 517 & \multirow[t]{4}{*}{0.21} \\
\hline N1 & 22 & 154 & \\
\hline $\mathrm{N} 2$ & 28 & 134 & \\
\hline N3 & 40 & 163 & \\
\hline \multicolumn{4}{|c|}{ Pathological stage } \\
\hline I & 79 & 403 & \multirow[t]{4}{*}{0.006} \\
\hline II & 30 & 225 & \\
\hline III & 35 & 228 & \\
\hline IV & 36 & 112 & \\
\hline
\end{tabular}

HER2 human epidermal growth factor receptor 2

Survival rates

After the median follow-up period of 62 months, the OS in 1,148 patients was analyzed to evaluate the prognostic impact of HER2 expression. HER2 expression scores obtained by IHC showed a distinct influence on survival, although patients with HER2 expression scores of both 1+ and $2+$ had similar survival curves (Fig. 1a). When we compared OS in terms of HER2 status, patients with HER2-positive gastric cancer showed much poorer survival than those whose tumors were HER2 negative [HR 1.59, $95 \%$ confidence interval (CI) 1.24-2.02; log-rank $P<0.001$ ] (Fig. 1b).

Subgroup analysis by pathological tumor stage showed a similar trend of poor survival in HER2-positive patients, although a higher HR for death was obtained in earlier stages, as follows: stage I, HR 2.04 (95 \% CI 1.14-3.65), 

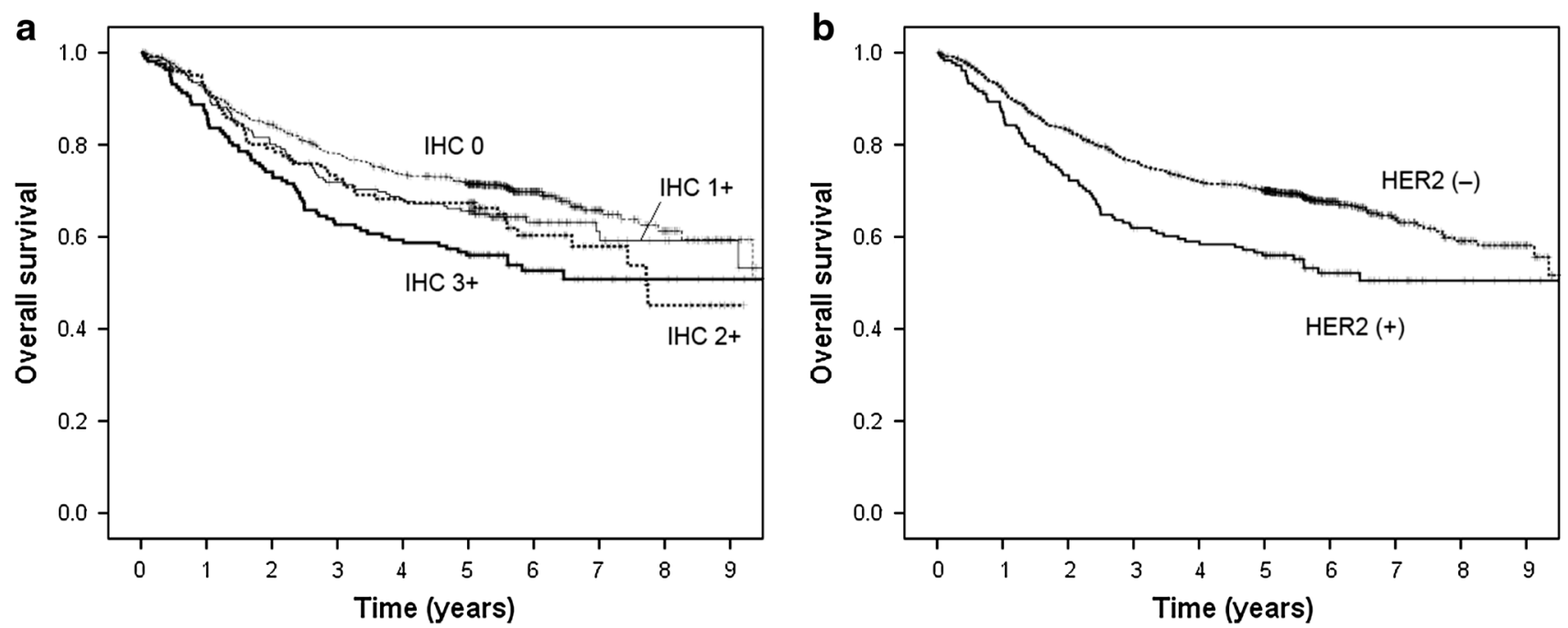

Fig. 1 Kaplan-Meier overall survival for all 1,148 patients according to a human epidermal growth factor receptor 2 (HER2) immunohistochemistry $(I H C)$ scores, and b HER2 overexpression status

$P=0.015 \quad$ (Fig. 2a); stage II, HR $1.89 \quad(95 \%$ CI 1.02-3.53), $P=0.041$ (Fig. 2b); stage III, HR 1.47 (95\% CI $0.95-2.28$ ), $P=0.082$ (Fig. 2c); and stage IV, HR 1.43 (95\% CI 0.95-2.16), $P=0.084$ (Fig. 2d). Regarding the histological type, both intestinal type and diffuse type showed significantly poor survival in HER2-positive patients [intestinal type, HR 1.70 (95\% CI 1.26-2.30), $P<0.001$; diffuse type, HR 1.88 (95\% CI 1.20-2.97), $P=0.005]$. HER2 positivity was also associated with significantly poorer survival both in patients who received adjuvant treatment and in those who did not [with adjuvant, HR 1.94 (95\% CI 1.34-2.80), $P<0.001$; without adjuvant, HR 1.46 (95\% CI 1.05-2.04), $P=0.023$ ].

Cox multivariate analysis of HER2 status and seven clinicopathological factors (age, sex, location, histological type, $\mathrm{pT}$, pN, adjuvant chemotherapy) revealed that age, $\mathrm{pT}$ stage, pN stage, and HER2 status were independent significant prognostic factors (Table 2). The adjusted HR for death in HER2-positive patients was 1.96 (95\% CI $1.51-2.55)$.

\section{HER2 heterogeneity}

Intratumoral heterogeneity of HER2 overexpression by IHC was evaluated for HER2-positive tumors. Of the 175 tumors that could be evaluated for heterogeneity, 132 (75.4\%) showed heterogeneous HER2 overexpression (Fig. 3). There were no homogeneous cases among the 18 tumors that had IHC scores of $2+$. Heterogeneous cases were significantly more likely to be classified as the diffuse type (Table 3). The distribution of pT stage also differed significantly between the homogeneous and heterogeneous groups. The OS in the heterogeneous group was similar to that in the homogeneous group (HR 0.88, $95 \%$ CI $0.54-1.45 ; P=0.63$ ) (Fig. 4).

\section{Discussion}

Our multicenter large-scale study of 1,148 patients demonstrated that HER2 overexpression was a significant factor associated with poor prognosis in patients with resectable gastric cancer. Differences in HER2 expression scores $(0 / 1+, 2+/ 3+)$ obtained by IHC showed a distinct influence on OS. HER2-positive patients with cancer of all stages had shorter survival than HER2-negative patients.

Although the prognostic impact of HER2 expression in patients with resectable gastric cancer has been previously examined in many small-scale retrospective studies, the prognostic value of HER2 is still controversial. A recent systematic review which included only studies with over 100 patients reported that most of the publications (71\%) showed that a HER2-positive status was associated with poor survival and/or clinicopathological characteristics, such as serosal invasion, lymph node metastases, or distant metastases [13]. Kim et al. [8] performed a tissue microarray analysis of HER2 expression in 595 Korean gastric cancer patients and found that HER2 overexpression was an independent prognostic factor in differentiated, resectable gastric cancer. On the other hand, Terashima et al. [10] reported no survival impact of HER2 expression in 829 Japanese gastric cancer patients. Several Japanese investigators might have considered this as a final answer to the long-debated issue, since the study analyzed a large number of archival specimens obtained in collaboration with the pivotal nationwide phase III trial. Curiously, the 

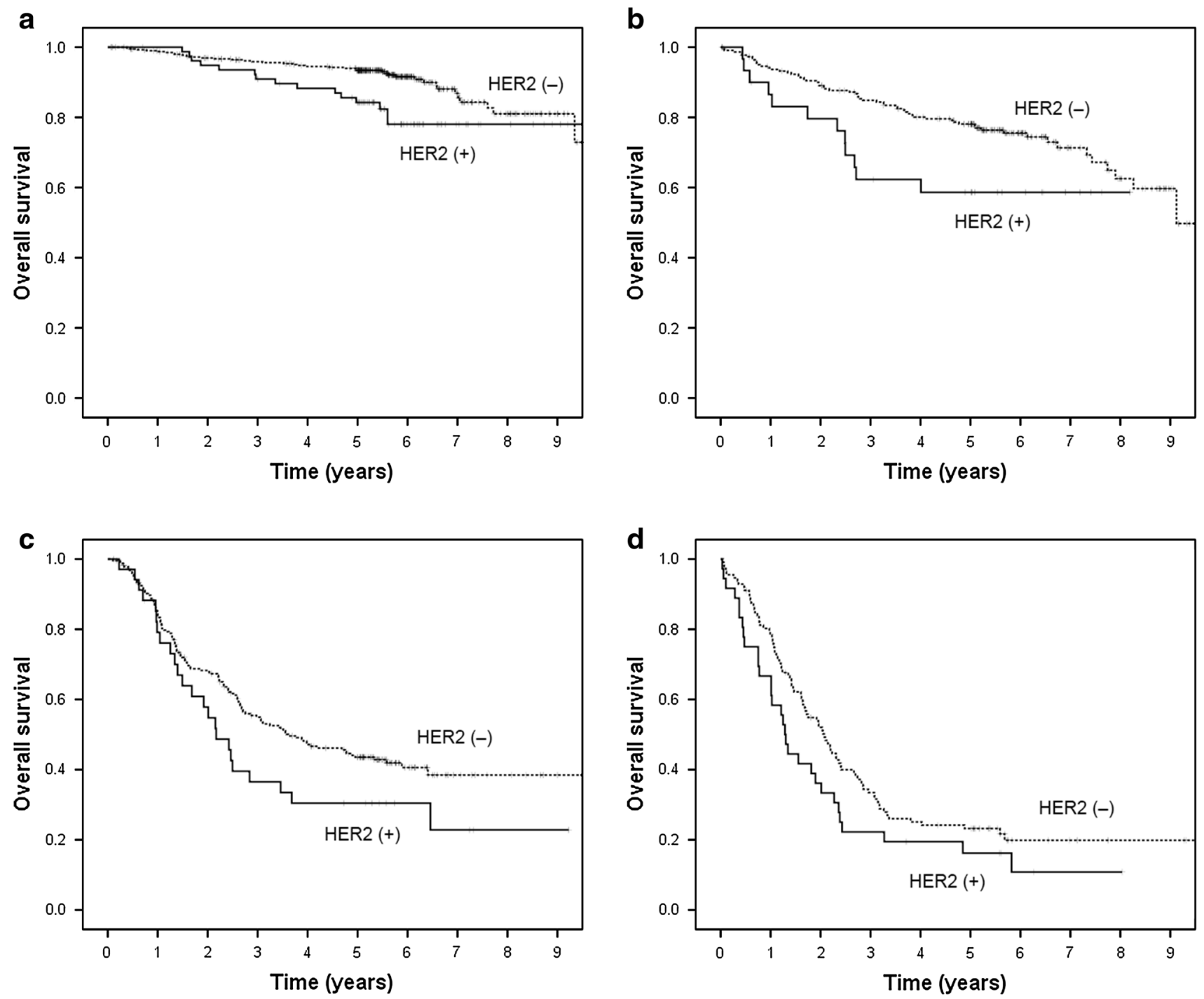

Fig. 2 Kaplan-Meier overall survival for patients with different stages of gastric cancer: a 482 patients with stage I cancer; $\mathbf{b} 255$ patients with stage II cancer; c 263 patients with stage III cancer; d 148 patients with stage IV cancer

Table 2 Multivariate overall survival analysis

\begin{tabular}{lllc}
\hline Variables & Category & HR $(95 \%$ CI $)$ & $P$ \\
\hline Age (years) & $\geq 70$ & $2.05(1.67-2.51)$ & $<0.001$ \\
Sex & Male & $1.23(0.97-1.54)$ & 0.085 \\
Location & Upper & $1.25(1.00-1.56)$ & 0.055 \\
Histological type & Diffuse type & $1.07(0.86-1.33)$ & 0.57 \\
pT & T3-4 & $3.23(2.44-4.27)$ & $<0.001$ \\
pN & N1-3 & $2.90(2.22-3.77)$ & $<0.001$ \\
Adjuvant chemotherapy & Yes & $1.24(1.00-1.55)$ & 0.055 \\
HER2 & Positive & $1.96(1.51-2.55)$ & $<0.001$ \\
\hline
\end{tabular}

CI confidence interval, $H R$ hazard ratio

HER2 positivity rate $(13.6 \%)$ in the study of Terashima et al. was relatively low compared with the rates obtained in other large-scale studies, although whether this explains

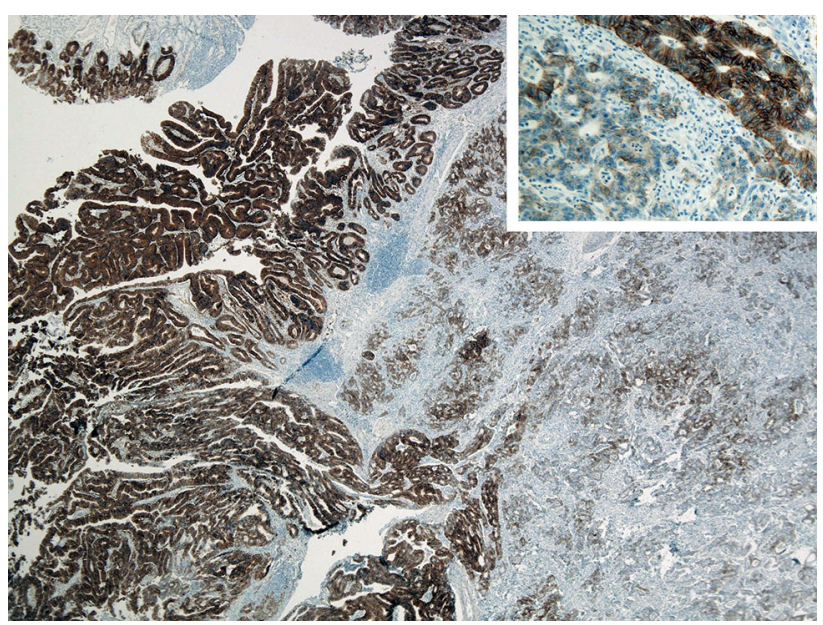

Fig. 3 Intratumoral heterogeneity of HER2 overexpression 
Table 3 Characteristics of 175 HER2-positive patients stratified by intratumoral HER2 heterogeneity

\begin{tabular}{|c|c|c|c|}
\hline Characteristics & $\begin{array}{l}\text { Homogeneous } \\
(n=43)\end{array}$ & $\begin{array}{l}\text { Heterogeneous } \\
(n=132)\end{array}$ & $P$ \\
\hline \multicolumn{4}{|l|}{ Age (years) } \\
\hline Median & 66 & 68 & \multirow[t]{2}{*}{0.27} \\
\hline Range & $46-80$ & $37-87$ & \\
\hline \multicolumn{4}{|l|}{ Sex } \\
\hline Male & 34 & 97 & \multirow[t]{2}{*}{0.46} \\
\hline Female & 9 & 35 & \\
\hline \multicolumn{4}{|l|}{ Location } \\
\hline Upper & 13 & 32 & \multirow[t]{2}{*}{0.44} \\
\hline $\begin{array}{c}\text { Middle/ } \\
\text { lower }\end{array}$ & 30 & 100 & \\
\hline \multicolumn{4}{|c|}{ Histological type } \\
\hline $\begin{array}{l}\text { Intestinal } \\
\text { type }\end{array}$ & 40 & 99 & \multirow[t]{2}{*}{0.011} \\
\hline Diffuse type & 3 & 33 & \\
\hline \multicolumn{4}{|l|}{ pT } \\
\hline $\mathrm{T} 1$ & 13 & 60 & \multirow[t]{4}{*}{0.008} \\
\hline $\mathrm{T} 2$ & 5 & 19 & \\
\hline $\mathrm{T} 3$ & 15 & 16 & \\
\hline $\mathrm{T} 4$ & 10 & 37 & \\
\hline \multicolumn{4}{|l|}{$\mathrm{pN}$} \\
\hline N0 & 17 & 69 & \multirow[t]{4}{*}{0.17} \\
\hline N1 & 6 & 16 & \\
\hline $\mathrm{N} 2$ & 11 & 16 & \\
\hline N3 & 9 & 31 & \\
\hline \multicolumn{4}{|c|}{ Pathological stage } \\
\hline I & 14 & 62 & \multirow[t]{4}{*}{0.32} \\
\hline II & 8 & 21 & \\
\hline III & 9 & 26 & \\
\hline IV & 12 & 23 & \\
\hline \multicolumn{4}{|c|}{ HER2 IHC score } \\
\hline $2+$ & 0 & 18 & \multirow[t]{2}{*}{0.011} \\
\hline $3+$ & 43 & 114 & \\
\hline
\end{tabular}

IHC immunohistochemistry

the difference in the results between the study of Terashima et al. and our study is unknown. The HER2 positivity rate in our study $(15.7 \%)$ was similar not only to that in the Western ToGA study (16.6\%), but also to that in a recent Japanese cohort study $(15.5 \%)[14,15]$.

Some studies have evaluated the prognostic impact of HER2 expression also in unresectable or recurrent gastric cancer. Janjigian et al. [16] conducted a retrospective multicenter study examining HER2 expression in 381 Western patients with metastatic gastric or esophagogastric junction cancer, and reported that HER2 overexpression was not an independent prognostic factor. Shitara et al. [17] conducted a retrospective study in Japanese patients with

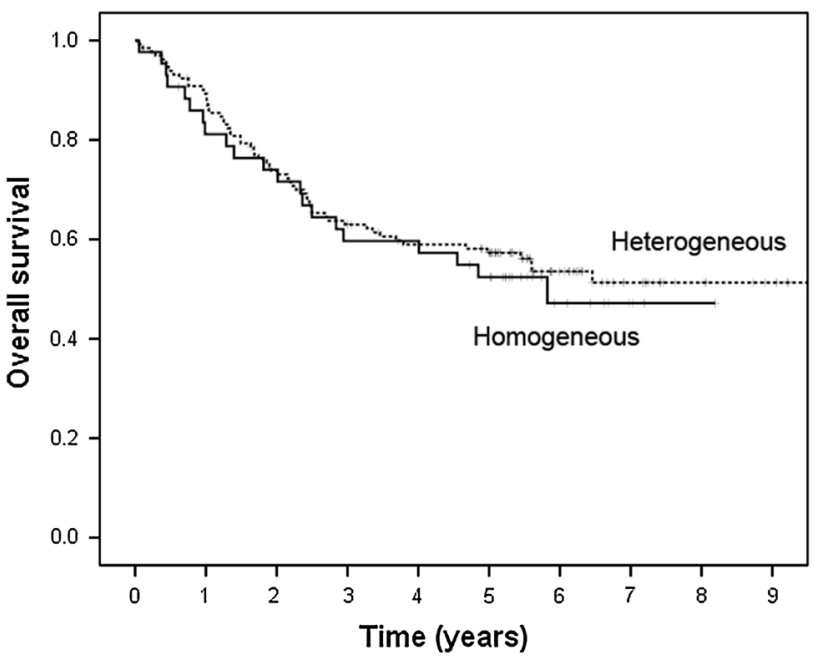

Fig. 4 Kaplan-Meier overall survival for 175 HER2-positive patients stratified by intratumoral HER2 heterogeneity

unresectable or recurrent gastric cancer, and reported that the OS in 15 HER2-positive patients not receiving trastuzumab treatment was similar to that in 306 HER2-negative patients. Recently, a Chinese prospective cohort study showed that 51 HER2-positive patients receiving trastuzumab had prognoses comparable to those of 251 patients with HER2-negative advanced gastric cancer, whereas 47 HER2-positive patients not receiving trastuzumab had the poorest prognosis [18]. Although our large-scale study demonstrated that HER2 expression is an independent prognostic factor in resectable gastric cancer, the prognostic value of HER2 status in unresectable or recurrent gastric cancer is still unknown. Indeed, there were smaller differences between HER2-positive and HER2-negative patients in the stage IV cancer subgroup than in the stage I cancer, stage II cancer and stage III cancer subgroups. We anticipate that an ongoing cohort study evaluating HER2 expression in patients with unresectable or recurrent gastric cancer will clarify this issue.

Intratumoral HER2 heterogeneity is important because it may lead to inaccurate assessment of HER2 status and the consequent inappropriate choice of trastuzumab treatment. It has been reported that HER2 heterogeneity is more frequent in gastric cancer than in breast cancer [11]. Indeed, intratumoral HER 2 heterogeneity by IHC was observed in three quarters of the HER2-positive tumors in our study. Lee et al.[19] reported that HER2 homogeneity conferred poorer survival than HER2 heterogeneity in a small-scale $(n=64)$ retrospective study. Our study also revealed that heterogeneity was more frequent in diffuse-type tumors and in tumors with and IHC score of $2+$, and that there was no impact on prognosis. However, the impact on trastuzumab response remains unclear, so further studies to evaluate the therapeutic impact of HER2 heterogeneity are warranted. 
To our knowledge, this is the first study to evaluate HER2 status in over 1,100 patients with gastric cancer. In conclusion, our multicenter large-scale study demonstrated that HER2 overexpression is an independent prognostic factor in gastric cancer patients. Although intratumoral HER2 heterogeneity was observed frequently, it did not affect prognosis at all.

Acknowledgments This work was supported by Chugai Pharmaceutical. The authors thank Y. Hayashi for her review of this report on behalf of Chugai Pharmaceutical.

\section{References}

1. Ferlay J, Shin HR, Bray F, Forman D, Mathers C, Parkin DM. Estimates of worldwide burden of cancer in 2008: GLOBOCAN 2008. Int J Cancer. 2010;127:2893-917.

2. Hudis CA. Trastuzumab-mechanism of action and use in clinical practice. N Engl J Med. 2007;357:39-51.

3. Seshadri R, Firgaira FA, Horsfall DJ, McCaul K, Setlur V, Kitchen P. Clinical significance of HER-2/neu oncogene amplification in primary breast cancer. J Clin Oncol. 1993;11:1936-42.

4. Dawood S, Broglio K, Buzdar AU, Hortobagyi GN, Giordano SH. Prognosis of women with metastatic breast cancer by HER2 status and trastuzumab treatment: an institutional-based review. J Clin Oncol. 2010;28:92-8.

5. Bang YJ, Van Cutsem E, Feyereislova A, Chung HC, Shen L, Sawaki A, et al. Trastuzumab in combination with chemotherapy versus chemotherapy alone for treatment of HER2-positive advanced gastric or gastro-oesophageal junction cancer (ToGA): a phase 3, open-label, randomised controlled trial. Lancet. 2010;376:687-97.

6. Wang YL, Sheu BS, Yang HB, Lin PW, Chang YC. Overexpression of c-erb-B2 proteins in tumor and non-tumor parts of gastric adenocarcinoma-emphasis on its relation to $\mathrm{H}$. pylori infection and clinicohistological characteristics. Hepatogastroenterology. 2002;49:1172-6.

7. Begnami MD, Fukuda E, Fregnani JH, Nonogaki S, Montagnini AL, Da Costa WL Jr, et al. Prognostic implications of altered human epidermal growth factor receptors (HERs) in gastric carcimomas: HER2 and HER3 are predictors of poor outcome. J Clin Oncol. 2011;29:3030-6.
8. Kim KC, Koh YW, Chang HM, Kim TH, Yook JH, Kim BS, et al. Evaluation of HER2 protein expression in gastric carcinomas: comparative analysis of 1,414 cases of whole-tissue sections and 595 cases of tissue microarrays. Ann Surg Oncol. 2011;18: 2833-40.

9. Kataoka Y, Okabe H, Yoshizawa A, Minamiguchi S, Yoshimura $\mathrm{K}$, Haga $\mathrm{H}$, et al. HER2 expression and its clinicopathological features in resectable gastric cancer. Gastric Cancer. 2013;16: 84-93.

10. Terashima M, Kitada K, Ochiai A, Ichikawa W, Kurahashi I, Sakuramoto $\mathrm{S}$, et al. Impact of expression of human epidermal growth factor receptors EGFR and ERBB2 on survival in stage II/ III gastric cancer. Clin Cancer Res. 2012;18:5992-6000.

11. Hofmann M, Stoss O, Shi D, Büttner R, van de Vijver M, Kim W, et al. Assessment of a HER2 scoring system for gastric cancer: results from a validation study. Histopathology. 2008;52:797-805.

12. Sobin LH, Gospodarowicz MK, Wittekind CH. TNM classification of malignant tumours. 7th ed. New York: Wiley-Blackwell; 2009.

13. Jørgensen JT, Hersom M. HER2 as a prognostic marker in gastric cancer-a systematic analysis of data from the literature. J Cancer. 2012;3:137-44.

14. Chung HC, Bang YJ. Human epidermal growth factor receptor 2 (HER2) in gastric cancer (GC): results of the ToGA trial screening programme and recommendations for HER2 testing. EJC Suppl. 2009;7(2):364.

15. Nishikawa K, Chin K, Nashimoto A. Result of HER2 status in Japanese metastatic gastric cancer-prospective cohort study (JFMC44-1101). J Clin Oncol 2012;30:(Suppl 4):10.

16. Janjigian YY, Werner D, Pauligk C, Steinmetz K, Kelsen DP, Jäger E, et al. Prognosis of metastatic gastric and gastroesophageal junction cancer by HER 2 status: a European and USA International collaborative analysis. Ann Oncol. 2012;23:2656-62.

17. Shitara K, Yatabe Y, Matsuo K, Sugano M, Kondo C, Takahari D, et al. Prognosis of patients with advanced gastric cancer by HER2 status and trastuzumab treatment. Gastric Cancer. 2013;16:261-7.

18. Qiu MZ, Li Q, Wang ZQ, Liu TS, Liu Q, Wei XL, et al. HER2positive patients receiving trastuzumab treatment have a comparable prognosis with HER2-negative advanced gastric cancer patients: a prospective cohort observation. Int J Cancer. 2014;134:2468-77.

19. Lee HE, Park KU, Yoo SB, Nam SK, Park DJ, Kim HH, et al. Clinical significance of intratumoral HER2 heterogeneity in gastric cancer. Eur J Cancer. 2013;49:1448-57. 